\title{
Forecasting fire dynamics using inverse Computational Fluid Dynamics modelling and Tangent Linearisation
}

\author{
W. Jahnª, G. Rein ${ }^{\text {a, }}$, J.L. Torero ${ }^{\mathrm{a}}$ \\ ${ }^{a}$ School of Engineering, The University of Edinburgh, \\ King's Buildings, Edinburgh, EH9 3JL, UK.
}

\begin{abstract}
A technology able to forecast fire dynamics in buildings would lead to a paradigm shift in the response to emergencies, providing the Fire Services with essential information about the ongoing blaze with some lead time (i.e. seconds or minutes ahead of the event). But the state-of-the-art of Computational Fluid Dynamics in fire dynamics is not fast nor accurate enough to provide valid predictions on time. This paper presents a methodology to forecast fire dynamics using Computational Fluid Dynamics based on assimilation of sensor observations. The forecast is posed as an inverse problem to solve for the invariants governing the dynamics, and a tangent linear approach is used in the optimisation. The forward fire model is linearized in order to obtain a quadratic cost function that is easily minimised. A series of real-scale compartment fire cases are investigated using the Computational Fluid Dynamics code FDSv5 together with synthetic data. Up to three different invariant are considered (spread rate, burning rate and soot yield) in scenarios with one or two fires and different origins. The effect of density, location and type of sensors is studied. It is shown that the use of coarse grids in the forward model significantly accelerates the assimilation up to 100 times without loss of forecast accuracy due to the aid of sensor data. This provides close to positive lead times using Computational Fluid Dynamics. These results are a fundamental step towards the development of forecast technologies able to lead the fire emergency response.
\end{abstract}

Keywords: Fire modelling, forecast, data driven simulation, gradient based optimization, tangent linear

\section{Introduction}

Forecasting compartment fire dynamics is a subject of research interest in fire safety science. After prevention, the first line of response to a fire event is part of the design process of a building in the form of detection, compartmentation, ventilation, egress paths, suppression and structural resistance ${ }^{1}$. But accidental fires that overcome these measures occur with some frequency, and it is necessary to prepare for that case and protect life and property against the detrimental effects of heat and smoke produced. When a fire escalates, intervention of the Fire Service takes place and management of the scene is delegated to them. The fire fighting strategy to follow is currently based mostly on the experience and the intuition of the commanding officers on duty. This offers space for improvement. In particular, it would be a great advantage if decisions could be based as well on short- and medium-term forecasts of the fire development. Not only would this allow for more efficient strategies, saving lives and reducing damage and cost, it would also improve the safety of fire fighters. The tragic events on 9/11 after the attacks of the World Trace Center are an example where prior knowledge of fire development and structural response would have been essential for the Fire Service.

Modern buildings already provide some limited information about an ongoing fire emergency, including security cameras and security panels which could indicate in a rudimentary manner the origin and magnitude of the event. The currently low density and crude nature of buildings sensor data, however, makes reliance on experience and intuition for fire fighting unavoid$\mathrm{able}^{2}$. In the near future, the role of intelligent building systems is expected to be central for the modern built environment. These systems are designed to monitor with sensors and control the performance of mechanical and electrical elements, and can easily be extended to include fire safety ${ }^{3}$.

Cowlard et al. ${ }^{2}$ have suggested the use of fire model predictions to assist emergency response. They postulate that the forecasting of fire dynamics in compart- 
ments would imply a paradigm shift in the response to emergencies, providing the Fire Service with essential information about the emergency development with some lead time (ahead of the event).

If computational models are ever to forecast fire in support of emergency response, the computational time has to be shorter than the event itself. From a fire fighting point of view, forecasting of fire only makes sense if it arrives with a positive lead time, i.e. data is assimilated and a satisfactory prediction is produced faster than the fire is evolving. But the state-of-the-art of computational fluid dynamics (CFD) for fire demands heavy computational resources and large computing times that are far greater than the times associated with fire dynamics (hours to model seconds). Moreover, the accuracy of the computer predictions of fire is poor due to the complex nature of the governing physical and chemical processes $^{4,5}$.

\section{Inverse Modelling}

Even if all the governing mechanisms could be solved from first principles, the chaotic nature of fire imposes a maximum possible lead time to the forecast, as has been shown for other dynamic systems like weather ${ }^{6}$. This suggests that fire modelling alone cannot be used for emergency response without feedback information from the evolving fire. Moreover, models complemented with sensor data have the potential to achieve the required speed, precision and robustness ${ }^{7}$. It has been proposed to use sensor data as a substitute for modelling fine details, thus enabling simpler approaches to provide fast and useful outputs ${ }^{8}$. Continuous correction of the model output by means of sensor data allows for steering of the models to account for uncertainty in the input variables and for changes in the environment (e.g. window breakage or flashover). This concept is illustrated in fig. 1, where sensor data is continuously assimilated in time to simulate the fire evolution and steer the computations.

The use of sensor data to estimate model parameters is generally known as inverse modelling. Parameter estimation and inverse modelling are an important area within the engineering and scientific community.

In numerical weather predictions (NWP) inverse modelling and data assimilation take up an important part of the computational resources ${ }^{10}$. In order to initialize the numerical models that are used to forecast the state of the atmosphere, weather observations from several places over the planet are collected and then assimilated into the model. Observations are assimilated

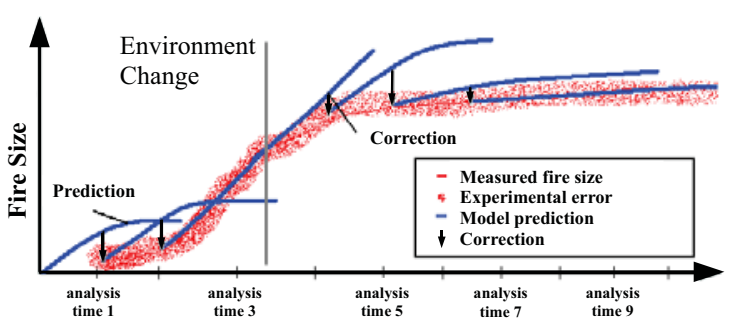

Figure 1: Conceptual representation of the data assimilation process and the sensor steering of model predictions even when fundamental changes take place in an evolving emergency scenario ${ }^{9}$.

by initializing a new forecast based on a previous forecast (several hours back), and correcting it according to the difference between the previous forecast and the observations ${ }^{11,12}$. Most modern NWP systems assimilate observations during a certain period of time (assimilation window) before starting the forecast ${ }^{13}$. The assimilation algorithm typically involves a linearization of the NWP system around the previous forecast and the subsequent solution of a quadratic minimization problem ${ }^{10}$. The basic principles of this methodology, called the tangent linear model (TLM), are applied in this work, adjusted to the specific features of fire dynamics.

In fire science, several inverse modelling studies have been undertaken. Richards et al. ${ }^{14}$ use a database of prerun zone-type models to estimate fire location and fire growth. They use the same zone model to generate the data for comparison, and analyze the influence of measurements and modelling errors. Leblanc and Trouvé use a zone model to predict the heat release rate (HRR) time-history based on observations generated using the same model ${ }^{15}$. While they are able to closely reproduce the HRR history, their work does not address the forecasting of future events.

Cowlard et al. ${ }^{7}$ use a semiempirical correlation and multiple flame front observations to predict upward flame spread over a small-scale fuel slab. They were able to estimate correctly the parameter values and make predictions in super-real-time (i.e. with a positive lead time). Koo et al. ${ }^{16}$ used measurements from a large-scale fire test to progressively steer zone model simulations towards effective parameter values using a Monte Carlo approach and a set of random generated scenarios. The model is able to reproduce satisfactorily past observations of temperature.

A conceptual framework for forecasting of fire growth has been proposed by $\mathrm{Jahn}^{9}$. The forecast is posed as an inverse problem to solve for the invariants 
governing the dynamics, and a minimisation technique is used. It has been applied to compartment fire using a zone model ${ }^{8}$, which rapidly estimated the correct invariants, but the forecasts were limited by the generalizations of the simple model. The present paper builds upon previous work and applies inverse modelling using CFD. The forward fire model (FDSv5) is linearized in order to obtain a quadratic cost function that is easily minimized. In order to demonstrate the performance of the approach, a series of realistic compartment fire cases are investigated. Further application of CFD forecasting to a real fire experiment is shown in ${ }^{17}$. These results are a fundamental step towards the development of forecast technology able to lead the fire emergency response.

In this document a conceptual framework and a mathematical methodology is proposed to allow for forecasting of fire growth. The highly complicated interaction between gas and solid phase are replaced by a simplified fire growth model that is input into the gas phase model as a boundary condition. This growth model is based on a set of parameters that do not depend on the feedback from the fire and are thus constant (at least for a certain amount of time). Observations from the evolving fire provide the information for the estimation of the parameters for the growth model.

\section{Fire Dynamics}

Natural fires in real-size compartments involve mechanisms that develop in length-scales ranging from micrometres (flame thickness) to metres (compartment size), and time-scales from milliseconds (chemistry) to minutes (burnout) ${ }^{1}$. Fire dynamics are governed by complex, strongly coupled physical and chemical processes constituting a feedback cycle. Pyrolysis vapours are produced in the thermal decomposition of the solid fuel as a result of heat transferred from the flame. The pyrolyzate is then transported into the flame where it mixes with fresh oxygen and burns. This results in heat that is transferred back to the fuel to produce more pyrolyzate $^{1}$.

Two fundamentally distinct regimes can be observed in a compartment fire. Each has to be treated differently. First a fuel controlled regime, and then a ventilation controlled regime (pre- and post-flashover respectively). The difference between both stages is illustrated in fig. 2. During the initial fuel controlled regime, sufficient air supply is available to feed the fire, and its spread rate is not governed by the ventilation, but by fuel load and arrangement. Boundary conditions are important only in the proximity of the flames, and the size and detailed geometry of the compartment does not greatly affect the course of the fire. For good forecast, modelling emphasis must be on the growth rate and the flame geometry.

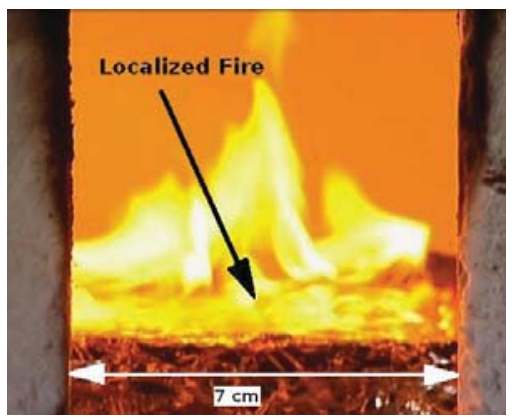

(a) Fuel controlled fire

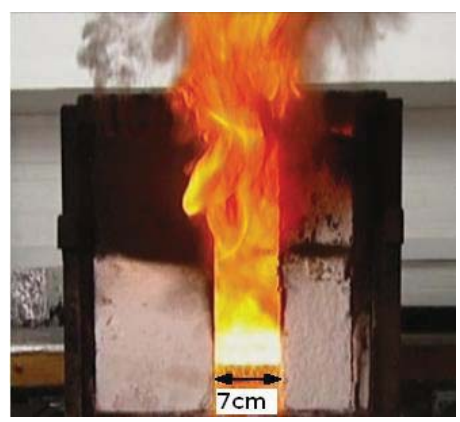

(b) Ventilation controlled fire

Figure 2: Different stages of the fire. a) shows a fuel controlled, localized fire, while b) shows a ventilation controlled fire.

As the fire grows beyond certain size, the compartment boundaries and the ventilation conditions start playing an essential role and the fire becomes controlled by the supply of air. At some point flashover will occur, after which external flaming takes place at the openings (doors, windows), as shown in fig. 2b. In a ventilation controlled regime, good forecasts require emphasis on the ventilation conditions and fire growth becomes secondary.

This work is focused on the fuel controlled regime during which the flame spread rate is the primary invariant to estimate growth rate.

\section{Fire Modelling}

In fire CFD modelling, the gas-phase dynamics are solved with a specific form of Navier-Stokes for buoyancy driven, low Mach number flows ${ }^{18,19}$. This is typically coupled with a mixture fraction model for combustion. Smoke movement and temperature distributions 
can only be reproduced reasonably well if the curve of HRR is known ${ }^{4,20,21}$.

Fire modelling typically handles compartment length scales of around $10 \mathrm{~m}$. Although depending on the size of the fire, with a large eddy simulation (LES) approach a grid resolution between $1 \mathrm{~cm}$ and $10 \mathrm{~cm}$ in the gas phase is generally required to obtain sufficient spatial resolution ${ }^{19}$. Thus, the computational cost of modelling fire is considerable.

The fire growth is given by the HRR which is governed by the spread of the flames over the solid fuel. While flame spread can be reproduced with numerical models to considerable accuracy for small flames $(\sim 10 \mathrm{~cm})$ by solving for the pyrolysis of the solid fuel ${ }^{22,23}$, it is much less accurate and computationally more expensive to do so in compartment, as the domain typically consists of dozens of metres, but grid cells of the order of millimetres would be required for the modelling of the flaming and pyrolizing regions. A recent study has shown that flame spread modelling is not satisfactorily at a scale that can be used in real scale fire scenarios $^{5}$, even using a relatively fine grid $(2.5 \mathrm{~cm})$. Flame spread and fire growth modelling are subject to ongoing research and have not been implemented satisfactorily yet ${ }^{4}$.

The limitations of state-of-the-art fire growth modelling are thus an important constraint to the forecast capabilities of fire simulations. It is proposed in this article to decouple the gas phase from the solid phase by imposing the fire growth as a time dependant boundary condition to the gas phase modelling. The interaction between gas and solid phase is replaced by a simplified fire growth model, which is explained in the following section.

\section{Fire Growth}

It can be assumed that the radius of the burning area (fire size) of an isotropic fuel grows at a constant rate. This is a reasonable assumption for early fire development when flames do not penetrate into the smoke layer and radiative heat from the walls do not accelerate spread $^{24}$. The fire source becomes thus a time dependent boundary condition to the gas phase simulation. Assuming a horizontal fire that starts at one point and spreads outwards radially with a spread rate $r(\mathrm{~m} / \mathrm{s})$, and assuming a constant fuel burning rate per unit area $\dot{\omega}\left(\mathrm{kg} / \mathrm{s} \cdot \mathrm{m}^{2}\right)$, the rate of heat release becomes proportional to the area of the fire, which grows as a function of the spread rate and of time,

$$
\dot{Q}=\Delta h_{c} \dot{m}=\Delta h_{c} \dot{\omega} A(r, t),
$$

where $\Delta h_{c}$ is the effective heat of combustion (in $\mathrm{kJ} / \mathrm{kg}$ ). As long as the fire does not reach the boundaries of the fuel surface the fire area is circular $(A(r, t)=\pi(r(t-$ $\left.\left.t_{0}\right)\right)^{2}$ ). Or it could have form of a fraction of a circle, if the ignition point is e.g. at the wall. In both cases the resulting HRR follows a quadratic growth.

This is a first approximation to simulate fire growth. It avoids direct coupling of gas and solid phase. A similar approach is widely used in fire safety engineering, although the governing parameters are summarized into a constant $\alpha$ that is tabulated for different materials and leads to what is known as an " $\alpha t^{2}$ " fire.

For real fuel packages (such as sofas, beds or other furniture), which consist of several finite surfaces each with a potentially different spread rate, this approximation has shown to still hold to a reasonable degree ${ }^{25}$, and the constant $\alpha$ corresponds in that case to an equivalent growth rate.

In fire field models the spread rate $r$ can be prescribed, so that adjacent cells are ignited producing a fire area that grows at rate $r$. This will then result in a fire that grows according to eq. (1).

\section{Inverse Modelling}

\subsection{Framework}

In order to be able to predict the state of a physical system, it is necessary to identify a set of parameters that characterize the system, and that do not change over time (or change only due to external intervention). These parameters are the invariants of the system, and a good forecast relies on good a estimation of these. A typical forecast cycle thus includes a data collection period, an assimilation period where the invariants are estimated, and finally a forecast based on those estimated parameters.

When modelling a physical process from first principles, the initial and boundary conditions constitute the only invariants, and changes of these conditions are only due to external intervention (for example a periodic boundary condition). In that case the invariants are well established. However, physical systems cannot generally be modelled from first principles, and approximations have to be introduced, resulting in additional invariants that have to be input into the model. These invariants are normally obtained experimentally.

In the present case the pyrolysis process is replaced by a fire growth model which results in a boundary condition that changes over time according to eq. (1). The invariants of interest are the spread rates of the burning items, the fuel burning rates and other parameters such 
as soot yield, radiative fraction (related to other approximations in the model).

The problem can thus be represented on the basis of these invariants summarized in the vector $\boldsymbol{\theta}$ :

$$
\boldsymbol{\theta}=\left[r_{1}, r_{2}, \ldots, \dot{\omega}_{1}, \omega_{2}, \ldots, \chi_{R, 1}, \chi_{R, 2} \ldots\right]
$$

Once a fire has been detected and observations are collected during the assimilation window, the relevant invariants in $\boldsymbol{\theta}$ are estimated and a forecast of the fire development is made without solving the complex interactions between gas and solid phases. The assimilation window is the period of time where observations are received and considered for the optimisation step. As time goes by, new observations come in, providing more information on the history of the fire development. In the present work the term observation refers to sensor data such as temperature or smoke obscuration.

The methodology is general and any forward model that represents the system to be simulated can be used. For scenarios described in this work and the level of detail required in the approach presented in this article, a CFD type fire model is used.

\subsection{Cost Function}

Data are assimilated into the model by minimizing a cost function that measures the distance between the model output and the observations. The governing parameters are then adjusted until convergence is obtained. Several different physical observations can be used, but temperature and smoke obscuration are used here because they are the easiest to measure satisfactorily with current technology. The cost function is then defined as the sum of the distances between model output for a given set of parameters and the measurements:

$$
J(\boldsymbol{\theta})=\sum_{i}^{N}\left[\mathbf{y}_{i}-\hat{\mathbf{y}}_{i}(\boldsymbol{\theta})\right]^{T} \mathbf{W}_{i}\left[\mathbf{y}_{i}-\hat{\mathbf{y}}_{i}(\boldsymbol{\theta})\right],
$$

where $\mathbf{y}_{i}$ is the set of physical variables that is measured, and $\hat{\mathbf{y}}_{i}(\boldsymbol{\theta})$ is the output of the forward integration of the numerical model that computes the state of the system at time $t_{i}$ from the initial state $\mathbf{y}_{0}$, and $\mathbf{W}_{i}$ is a weight matrix. Data is assimilated during the assimilation window that is discretized according to the output of the numerical model in $N$ time steps.

The weight matrix $\mathbf{W}_{i}$ is used to assign different weights to each observation. These generally reflect trustworthiness of the measurements and are obtained from statistical analysis ${ }^{10}$. In the context of this paper $\mathbf{W}_{i}$ was considered to be the identity matrix, as all sensor data are considered equality reliable.
The inverse problem that has to be solved in order to estimate the parameters $\boldsymbol{\theta}$ can be formulated as the following minimization problem:

$$
\begin{array}{cl}
\min _{\boldsymbol{\theta}} & J(\boldsymbol{\theta}) \\
\text { s.t. } & \hat{\mathbf{y}}_{i}(\boldsymbol{\theta})=\mathcal{M}_{i}\left(\mathbf{y}_{0}, \boldsymbol{\theta}\right),
\end{array}
$$

where $\mathcal{M}_{i}\left(\mathbf{y}_{0}, \boldsymbol{\theta}\right)$ denotes the forward integration model (a fire specific CFD code in this case).

\subsection{Optimisation}

Several methods can be used to minimise the non linear cost function $J(\boldsymbol{\theta})$, which can be summarized in two groups: gradient based and gradient-free methods. Gradient-free methods are heuristic methods that include a random component in the search and evolve towards the global minimum following different laws of selection (for example survival of the fittest in Genetic Algorithms) combined with a stochastic component. Gradient methods start from an initial guess relatively close to the minimum and then use information of the gradient to establish a search direction and a step size. One important advantage of gradient-free methods is they are very robust regarding the objective function to minimize and do not require smoothness or continuity. However, they tend to need a large amount of function evaluations in each iteration and have slow convergence rates compared to gradient based methods ${ }^{26}$. In minimization problems where the cost function is continuous and an initial guess can assured to be in the vicinity of a global minimum, gradient based optimization methods outperform evolutionary methods in terms of number of evaluations and convergence speed ${ }^{26,27}$.

The continuity of $J(\boldsymbol{\theta})$ and the relatively narrow range of possible parameters (spread and burning rates are within limited ranges) provided by access to laboratory data in the literature, suggest the use of gradient based methods. Furthermore, the high cost of function evaluation (each evaluation of $\boldsymbol{J}(\boldsymbol{\theta})$ involves a forward integration of the fire model) makes evolutionary algorithms unattractive.

The computation of the gradient of the cost function (eq. (2)) involves the differentiation of the forward model with respect to the parameters, as shown in eq. (4).

$$
\nabla J(\boldsymbol{\theta})=-2 \sum_{i}^{N} \nabla \mathcal{M}_{i}\left(\mathbf{y}_{0}, \boldsymbol{\theta}\right)^{T} \mathbf{W}_{i}\left[\mathbf{y}_{i}-\mathcal{M}_{i}\left(\mathbf{y}_{0}, \boldsymbol{\theta}\right)\right] .
$$

As a first approximation, a Finite Differences (FD) scheme was used to approximate the gradient of the for- 
ward model,

$$
\frac{\partial \mathcal{M}_{i}\left(\mathbf{y}_{0}, \boldsymbol{\theta}\right)}{\partial \theta_{j}} \simeq \frac{\mathcal{M}_{i}\left(\mathbf{y}_{0}, \boldsymbol{\theta}+\boldsymbol{\varepsilon}_{j}\right)-\mathcal{M}_{i}\left(\mathbf{y}_{0}, \boldsymbol{\theta}\right)}{\left\|\boldsymbol{\varepsilon}_{j}\right\|},
$$

where $\boldsymbol{\varepsilon}_{j}$ is a vector with a small perturbation in $\theta_{j}$. While this is very easy to implement, the accuracy of the derivative depends on the size of the perturbation $\boldsymbol{\varepsilon}_{j}$. Note that this approach rapidly becomes computationally expensive when a large set of parameters has to be estimated, as it requires an additional run of the forward model for each parameter. It is, however, computationally cheap for small sets like the one at hand ( $<5$ invariants). This approach is also easy to parallelize, as the forward integration can be launched with each perturbation on a different processor.

\subsection{Tangent Linear Model}

There are a number of gradient based algorithms with different approaches as to how to choose the best search direction and the most suitable step size. In the case of a linear forward model the cost function is quadratic, which can be minimized in one step by solving a linear system. While this is computationally cheap, physical systems tend not to be linear. For a non-linear forward model as the CFD one at hand, the tangent linear model (TLM) can be used ${ }^{28,10}$. The TLM method consists in linearizing the forward model $\mathcal{M}_{i}$ around some initial guess, so that the cost function is approximated as quadratic. An extensive analysis of the numerical efficiency is presented in ${ }^{10}$.

Let us consider a Taylor expansion of the forward model $\mathcal{M}_{i}$ around some initial guess $\boldsymbol{\theta}_{0}$,

$$
\mathcal{M}_{i}\left(\mathbf{y}_{0}, \boldsymbol{\theta}\right) \simeq \mathcal{M}_{i}\left(\mathbf{y}_{0}, \boldsymbol{\theta}_{0}\right)+\nabla \mathcal{M}_{i}\left(\mathbf{y}_{0}, \boldsymbol{\theta}_{0}\right)\left(\boldsymbol{\theta}-\boldsymbol{\theta}_{0}\right)
$$

The linearized forward model is then inserted into the cost function, yielding a quadratic function $\tilde{J}(\boldsymbol{\theta})$,

$$
\begin{gathered}
\tilde{J}(\boldsymbol{\theta})=\sum_{i=1}^{N}\left(\mathbf{y}_{i}-\left[\mathcal{M}\left(\mathbf{y}_{0}, \boldsymbol{\theta}_{0}\right)+\nabla_{\boldsymbol{\theta}} \mathcal{M}\left(\mathbf{y}_{0}, \boldsymbol{\theta}_{0}\right)\left(\boldsymbol{\theta}-\boldsymbol{\theta}_{0}\right)\right]\right)^{T} \\
\mathbf{W}_{i}\left(\mathbf{y}_{i}-\left[\mathcal{M}\left(\mathbf{y}_{0}, \boldsymbol{\theta}_{0}\right)+\nabla_{\boldsymbol{\theta}} \mathcal{M}\left(\mathbf{y}_{0}, \boldsymbol{\theta}_{0}\right)\left(\boldsymbol{\theta}-\boldsymbol{\theta}_{0}\right)\right]\right),
\end{gathered}
$$

where $\boldsymbol{\theta}$ is in the vicinity of $\boldsymbol{\theta}_{0}$.

The gradient of the quadratic cost function (eq. (7)) is then as follows

$$
\begin{aligned}
\nabla_{\boldsymbol{\theta}} \tilde{J}(\boldsymbol{\theta}) & =-2 \sum_{i=1}^{N}\left[\nabla_{\boldsymbol{\theta}} \mathcal{M}_{i}\left(\mathbf{y}_{0}, \boldsymbol{\theta}_{0}\right)\right]^{T} \mathbf{W}_{i} \\
& {\left[\mathbf{y}_{i}-\left(\mathcal{M}_{i}\left(\mathbf{y}_{0}, \boldsymbol{\theta}_{0}\right)+\nabla_{\boldsymbol{\theta}} \mathcal{M}_{i}\left(\mathbf{y}_{0}, \boldsymbol{\theta}_{0}\right)\left(\boldsymbol{\theta}-\boldsymbol{\theta}_{0}\right)\right)\right] . }
\end{aligned}
$$

Introducing the following annotation:

$$
\begin{aligned}
\mathbf{M}_{i} & =\mathcal{M}_{i}\left(\mathbf{y}_{0}, \boldsymbol{\theta}_{0}\right), \\
\mathbf{H}_{i} & =\nabla_{\boldsymbol{\theta}} \mathcal{M}_{i}\left(\mathbf{y}_{0}, \boldsymbol{\theta}_{0}\right), \\
\overline{\boldsymbol{\theta}} & =\left(\boldsymbol{\theta}-\boldsymbol{\theta}_{0}\right),
\end{aligned}
$$

it is possible to write the first order condition for optimality as follows:

$$
\sum_{i=1}^{N} \mathbf{H}_{i}^{T} \mathbf{W}_{i}\left[\mathbf{y}_{i}-\left(\mathbf{M}_{i}+\mathbf{H}_{i} \overline{\boldsymbol{\theta}}\right)\right]=0 .
$$

Equation (9) can be rearranged as

$$
\sum_{i=1}^{N} \mathbf{H}_{i}^{T} \mathbf{W}_{i} \mathbf{H}_{i} \overline{\boldsymbol{\theta}}=\sum_{i=1}^{N} \mathbf{H}_{i}^{T} \mathbf{W}_{i}\left(\mathbf{y}_{i}-\mathbf{M}_{i}\right),
$$

which is a linear system of the form

$$
\mathbf{A} \overline{\boldsymbol{\theta}}=\mathbf{b},
$$

where

$$
\begin{aligned}
\mathbf{A} & =\sum_{i=1}^{N} \mathbf{H}_{i}^{T} \mathbf{W}_{i} \mathbf{H}_{i}, \quad \text { and } \\
\mathbf{b} & =\sum_{i=1}^{N} \mathbf{H}_{i}^{T} \mathbf{W}_{i}\left(\mathbf{y}_{i}-\mathbf{M}_{i}\right) .
\end{aligned}
$$

By solving this linear system a new estimation of the parameters, $\widetilde{\boldsymbol{\theta}}^{*}$, is obtained. Note that the optimum of this problem is not necessarily equal to the optimum of the original minimization problem (eq. (3)). If the minimum $\boldsymbol{\theta}^{*}$ of the original minimization problem (eq. (3)) is close enough to the initial guess, the new estimate $\tilde{\boldsymbol{\theta}}^{*}$ will be equal to $\boldsymbol{\theta}^{*}$. If not, the procedure is repeated starting from this new point until convergence is obtained.

Figure 3 illustrates the inverse modelling process using the TLM approach. A scriptfile calls the forward model (CFD) jobs $(k+1$ in order to compute the $k$ partial derivatives for the gradient of $\mathcal{M}_{i}\left(\mathbf{y}_{0}, \boldsymbol{\theta}\right)$ ), and, when all of them have finished, calls the executable that computes the TLM. Each CFD job provides an output file with the data for the calculation of the gradient. These files are read by the TLM executable, which then computes $\overline{\boldsymbol{\theta}}$ by minimizing the TLM and provides a new set of input files for the next iteration. The process is repeated until a user specified convergence criterion is met (changes in the parameters from one iteration to the next smaller than a threshold value).

An alternative to the TLM method for unconstrained non-linear problems, the quasi-Newton BroydenFletcher-Goldfarb-Shanno (BFGS) method is one of the 


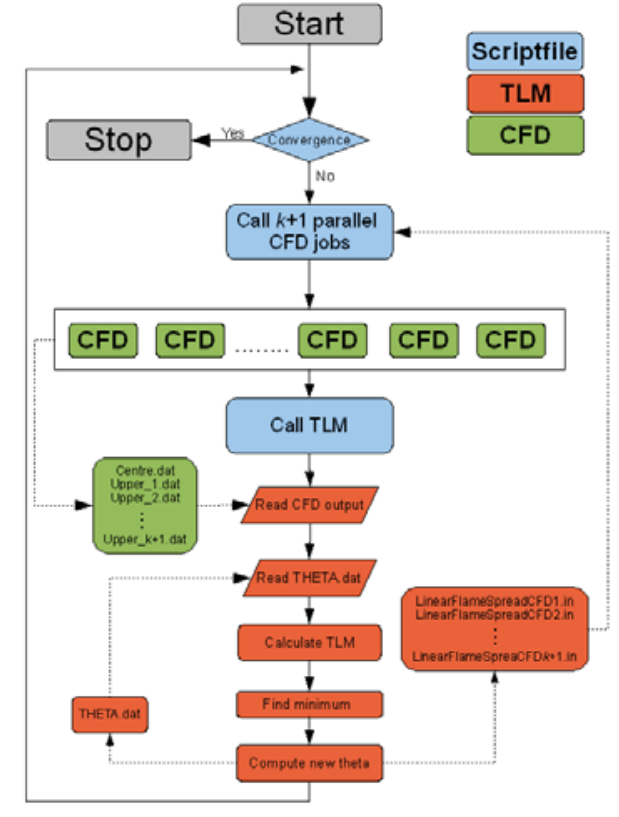

Figure 3: Inverse modelling procedure. The blue boxes indicate processes executed by a script file. Red boxes indicate the execution of the TLM method and its output files. Green boxes are the fire model.

most widely used as it requires only first order derivative computation, but conserves the good convergence characteristics of the Newton method ${ }^{27}$. The BGFS method is a Newton based optimization algorithm where the second order derivatives are estimated from the first order derivatives instead of calculating them directly. In spite of being computationally less expensive than the traditional Newton method, the computational effort of finding the optimum using the BFGS method is still considerable, as more than one function evaluation (model run) is required for each iteration in order to find an adequate step size.

Both the TLM and the BGFS methods were implemented in this work. It is shown in the results that TLM has a superior performance for the problem at hand.

\section{Results for a range of cases}

\subsection{Synthetic observations}

The inverse problem procedure explained in the previous sections is illustrated applying it to a range of fire cases. The CFD model used is the fire-specific LES code Fire Dynamics Simulator version 5.1.6 (FDSv5) ${ }^{29}$.

The test scenario consists of a compartment of $4 \mathrm{~m} \times$ $5 \mathrm{~m} \times 2.5 \mathrm{~m}$ with a door on one side, and a window on the opposite wall (see fig. 4). The fire is started at the corner of a bed located in the room as shown in fig. 5. It is then allowed to grow over the surface of the bed.

The observations used are synthetic data generated using the same CFD model (FDSv5) with a set of parameter values that are referred to as true values. For the spread rate, values reported in literature for real fires typically range from $1 \mathrm{~mm} / \mathrm{s}$ to $8 \mathrm{~mm} / \mathrm{s}$ depending on material and layout ${ }^{1}$. The range of possible values for the fuel mass flow rate is from 10 to $50 \mathrm{~g} / \mathrm{m}^{2} \mathrm{~s}^{1}$. The effective heat of combustion $\Delta h_{c}$ of the fuel is a relatively well defined quantity that depends mostly on the burning material and the combustion efficiency but varies only slightly for similar fuels ${ }^{30}$, and thus, an average value of $19 \mathrm{MJ} / \mathrm{kg}$ is used. It can be shown analytically that the HRR is most sensitive to the spread rate, followed by the fuel mass flow rate and is relatively insensitive to heat of combustion ${ }^{9}$. A grid size of $10 \mathrm{~cm}$ is used, and the synthetic observations are generated for $300 \mathrm{~s}$ of fire.

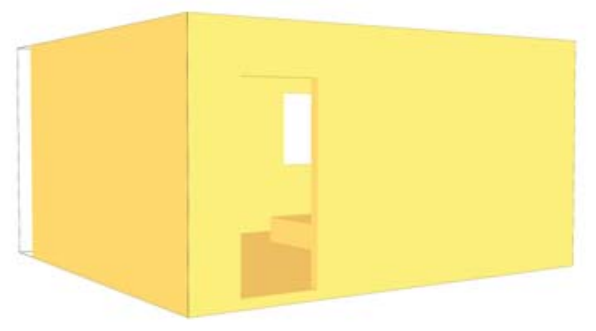

(a)

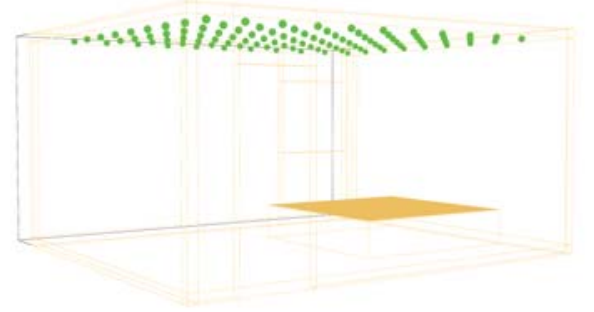

(b)

Figure 4: Set up for the fire scenario; a) the computational domain with the fire compartment (view through the door with the window on the opposite wall); b) the same compartment with outlined walls. The thermocouples in the ceiling are shown as dots.

The output of the CFD model, both for the forecast and for the observations, consists of wall temperatures 
measured with thermocouples in the ceiling and smoke obscuration from wall-to-wall beam detectors.

Wall temperature sensors are distributed uniformly on a rectangular grid throughout the ceiling, resulting in 99 sensor locations (approximate density of 5 sensors $/ \mathrm{m}^{2}$ ) as shown in fig. 5 . Eleven beam detectors across the compartment, installed $10 \mathrm{~cm}$ below the ceiling, provide smoke observation observations. The position of the full set of sensors is shown in fig. 5, where the blue lines represent the wall-to-wall laser beams crossing the room. White noise is added to the observations with a random, zero mean perturbation to the initial conditions, in order to account for realistic perturbations expected in sensor data.

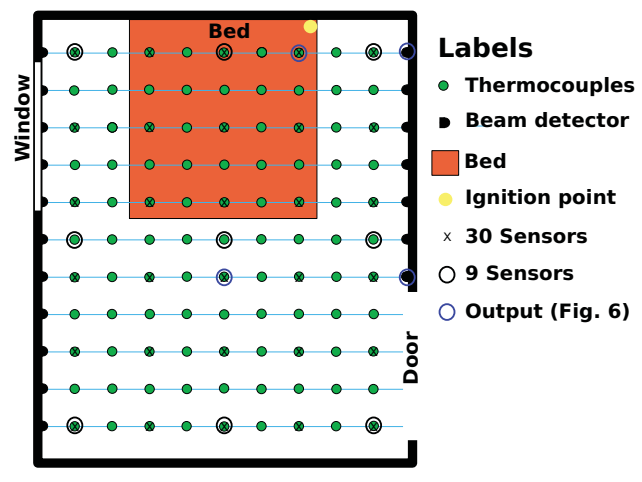

Figure 5: Top view of the compartment. The green dots represent the thermocouples in the ceiling, and the blue lines are the beam detectors.

Figure 6 shows a sample of sensor measurements generated in the true fire. The locations of the considered sensors are indicated by blue circles in fig. 5 . The time evolution of the ceiling temperature is shown for a thermocouple above the fire area (close to the ignition point) and for a thermocouple in an area towards the middle of the room, away from the fire. Additionally two examples of the percentage of obscuration as measured by a beam detector close to the ceiling are shown, one above the fire, and another away from the fire.

The forward model $\mathcal{M}$ considers the same geometry as the model used to generate the synthetic observations, including the position and dimensions of the bed and the grid size. The model is initialized with an arbitrary set of parameters (within physical limits). Note that the fire size follows a quadratic growth only as long as the fire does not reach the boundaries of the fuel surface (in this case the bed). Once the fire spreads to the bed edges, the growth would not be quadratic any more (power coefficient $<2$ ), since the spread rate is no longer constant

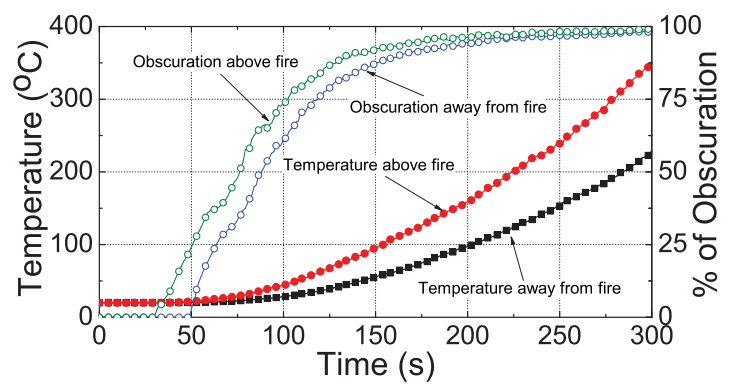

Figure 6: Sensor measurements recorded from the true fire.

in all directions.

\subsection{Single invariant}

First, a single invariant case is studied. Only the spread rate of the fire was estimated (true value of $2 \mathrm{~mm} / \mathrm{s}$ ), and the fuel burning rate was assumed known. The full set of ceiling temperature sensors is considered for assimilation. Observations are compared to model output directly, i.e. without prior data manipulation (no averaging or smoothing). Figure 7 compares the convergence of the spread rate of the bed fire obtained using the TLM to the convergence obtained using the BFGS minimization technique. Figure 7a shows that the BFGS method is slower to converge, and the final estimate differs significantly from the true value ( $>20 \%$ error). This is explained by the coarse approximation of the FD differentiation that is used to compute the gradient. If the estimated parameter at iteration $n$ is within a vicinity of the size of the perturbation used to compute the derivative, a descent direction cannot be assured, and the method is thus limited by the accuracy of the differentiation.

Using the TLM the convergence is faster and much more accurate $(<1 \%$ error). In this case the approximation of the gradient does not directly affect the precision of the estimation process, as it does not directly search for the zero of the gradient, but estimates the minimum based on assuming that the cost function can be approximated by a quadratic function close to the optimum. An additional advantage is that for the TLM only one model run is required for each parameter per iteration, whereas the BFGS method needs one run for each parameter and a few additional runs in each iteration to determine the step size that assures a descent. Note that the optimization process might produce negative numbers in early iterations (as shown in fig. 7a), which is not physical. In case this happens, the optimizer re-initializes the process in zero and starts over. 


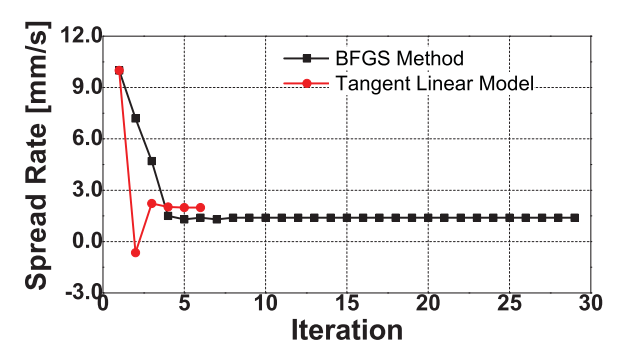

(a)

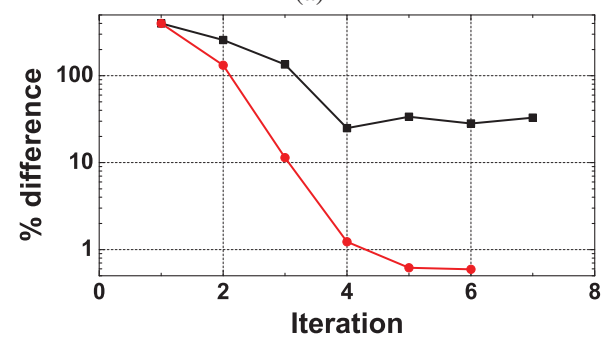

(b)

Figure 7: Comparison of TLM and BFGS for a single invariant problem; (a) shows the spread rate estimated at different iterations, and (b) shows the convergence of the error between the estimations and the true values.

Given the superior performance of the TLM, both in accuracy and in computation time, it was decided to concentrate on the TLM and not to develop the BFGS method for multi-parameter estimation.

\subsection{Two burning items}

A single burning item is the simplest case of a growing compartment fire. However, in many situations one item can ignite another close standing object when the flames engulf this object (for example a curtain can catch fire if it is close to a burning bed). The spread rates on both objects are not generally the same, and the capability of simultaneously estimating both spread rates was investigated here.

For this test case the same compartment explained in the previous section was simulated to generate the data, with a fire starting at the same corner of the bed as shown in fig. 5 , but simultaneously igniting wall lining material close by. One fire thus spreads horizontally over the bed as in the previous case, and a second fire is assumed to grow vertically upwards on the wall. In order to preserve the physical coherence of fire growth, the observations were generated using a faster growth for the vertical fire than for the horizontal $(1 \mathrm{~mm} / \mathrm{s}$ on the bed and $5 \mathrm{~mm} / \mathrm{s}$ on the wall). Note that a vertical fire will only grow at a constant spread rate as long as the flame is laminar ${ }^{31}$. Once the flame becomes turbulent,

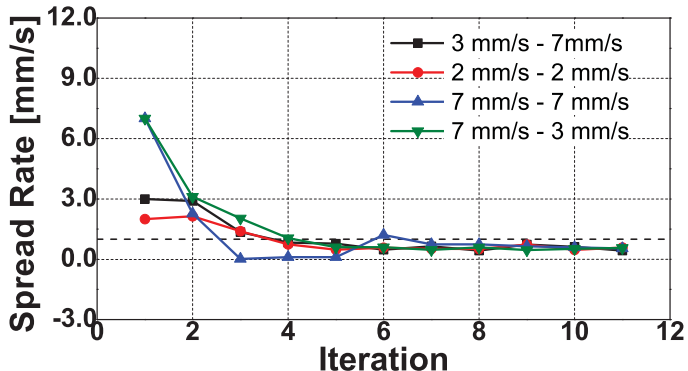

(a)

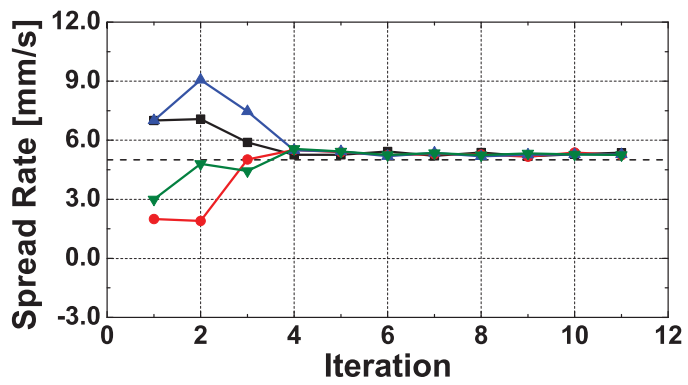

(b)

Figure 8: The spread rates for two independent fires are estimated using TLM for different first guesses. (legend gives values in sets of two, one for each spread rate). Graph a) shows the convergence of the spread rate on the bed, and $b$ ) shows the spread rate of the fire growing vertically on the wall. The dashed line marks the true value.

the spread rate starts accelerating, and the fire growth model used here (eq. (1)) no longer holds. Within the scope of this work, the vertical fire is for illustration purposes only, and a constant spread is assumed.

The forward model was initiated using different first guesses for the parameters. In fig. 8 the convergence of the estimated spread rates towards the true spread rates is shown. Each curve represents a pair of first guesses ranging from $2 \mathrm{~mm} / \mathrm{s}$ to $7 \mathrm{~mm} / \mathrm{s}$ (e.g. black square data was started with a spread rate of $3 \mathrm{~mm} / \mathrm{s}$ and $7 \mathrm{~mm} / \mathrm{s} \mathrm{re-}$ spectively). The estimation procedure is shown to be robust regarding the first guess (within the range of physically meaningful values). Figure 8 a shows that the horizontal spread rate is slightly underestimated. The vertical spread rate on the other hand is overestimated by around $7 \%$, as shown in fig. 8b. Although the spread rates do not agree exactly with the spread rates from the true fire, the difference remains within reasonable bounds. This demonstrates the overall robustness of the estimation method, as it could correctly differentiate between the two fires. 


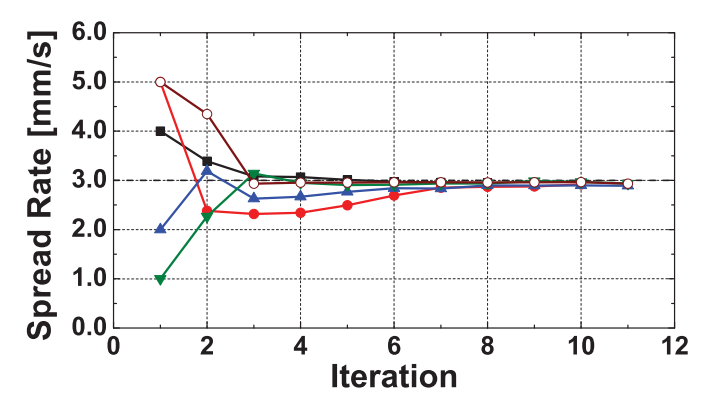

(a)

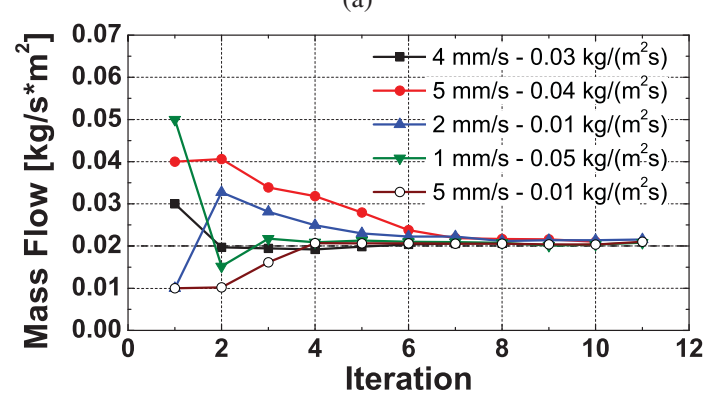

(b)

Figure 9: Estimation of spread rate and fuel mass flow rate for a single item burning. Graph a) shows the convergence of the burning rate, and b) shows the spread rate of the fire on the bed. The dashed line marks the true value.

\subsection{Single burning item and two invariants}

In this example it is attempted to solve for the fuel burning rate together with the spread rate for a single burning fire growing over the bed as in the single invariant case (section 7.2). The observations used for assimilation are generated using a true spread rate of $3 \mathrm{~mm} / \mathrm{s}$ and a true fuel burning rate of $0.02 \mathrm{~kg} / \mathrm{s} \cdot \mathrm{m}^{2}$ (typical of residential fuels ${ }^{30}$ ). The estimation process is initiated using different values ranging between 0.01 and $0.05 \mathrm{~kg} / \mathrm{s} \cdot \mathrm{m}^{2}$ for the fuel burning rate, and values between $1 \mathrm{~mm} / \mathrm{s}$ and $5 \mathrm{~mm} / \mathrm{s}$ for the spread rate.

The convergence of the spread rate and of the fuel burning rate of a single burning item is shown in fig. 9 . While good convergence is obtained for most first guesses after four iterations, in some cases the convergence is noticeably slower (eight iterations). The final difference between the true values and the estimations is of less than $3 \%$ for all initial guesses.

\subsection{Sensitivity to Sensor Locations}

Inverse problems by definition cannot assure a unique solution. The more information about the state of the system that can be obtained from the observations, the better the estimations of the parameters, since more information helps ruling out worse solutions. It was thus investigated how the density and distribution of sensors affects the outcome of the parameter estimation.

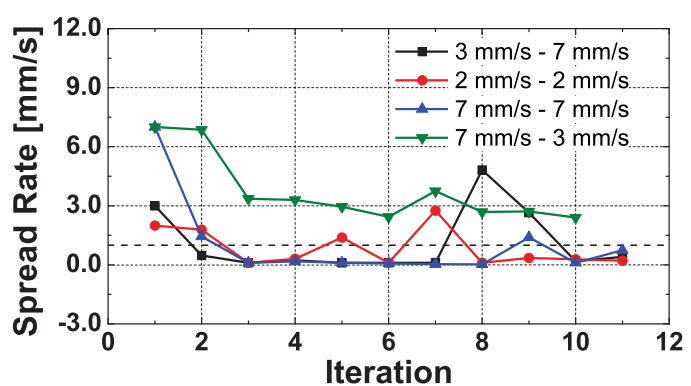

(a)

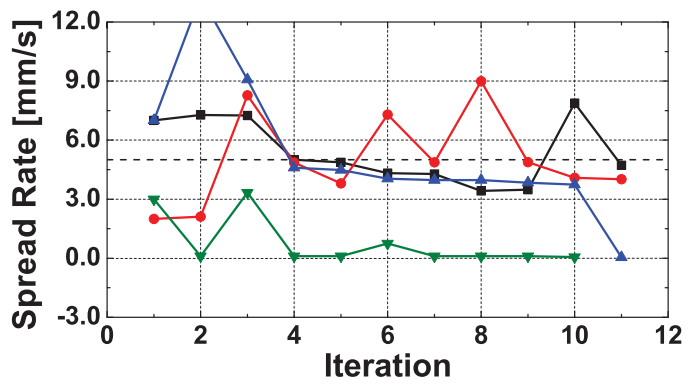

(b)

Figure 10: The spread rates for two items burning are estimated with only nine sensors. Graph a) shows the spread rate on the bed, and b) shows the spread rate of the wall fire. The dashed line marks the true value.

Figure 10 shows the estimation process for the parameters using the scenario of section 7.3 , with a fire on the bed and a second fire on the wall (same true values as before). Instead of the full set of sensors, the a subset of nine temperature sensors was used for assimilation, distributed uniformly throughout the ceiling as indicated in fig. 5, where the thermocouples that are considered are enclosed in a circle. It is clear from fig. 10 that nine sensors are not enough to differentiate between the two spread rates. Overall convergence of parameters is poor and slow.

Using 30 uniformly distributed sensor locations (the thermocouples marked with a cross in fig. 5, approximate density of 1.5 sensors $/ \mathrm{m}^{2}$ ) results in a better and faster estimation of the parameters as shown in fig. 11. Although convergence cannot be obtained for all first guesses in less than ten iterations, for those first guesses where the parameters converge fast, the estimations are within $15 \%$ of the true values.

Figure 12 shows the convergence history for the invariants in the case that only the sensors in the back 


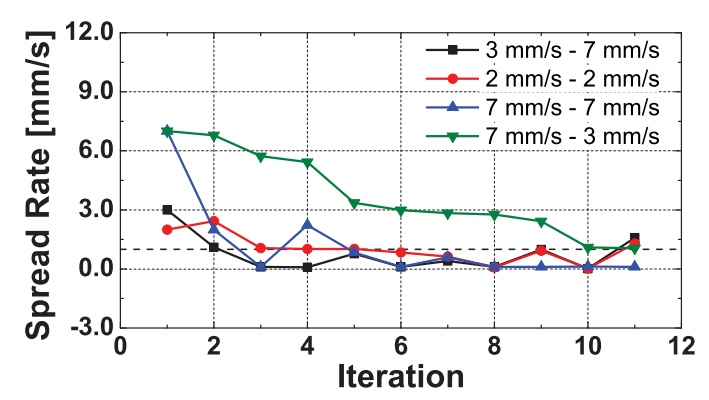

(a)

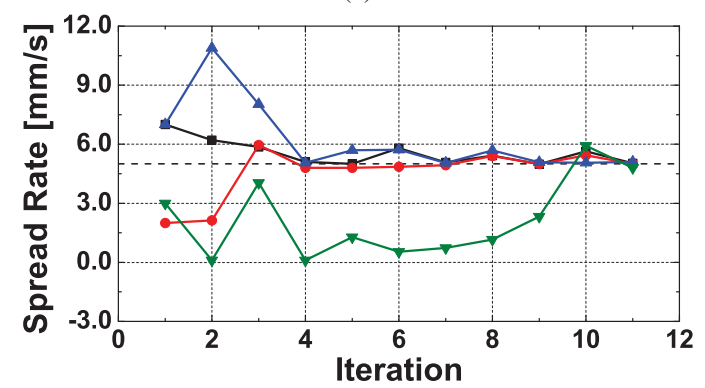

(b)

Figure 11: The spread rates of two items burning are estimated with only 30 sensors. Graph a) shows the spread rate on the bed, and b) shows the spread rate of the wall fire. The dashed line marks the true value.

part of the compartment, where the bed is located, are considered for the assimilation. The invariants are estimated more accurately than in the case where all sensors are considered (fig. 8). This is explained by the sensors away from the fire not providing useful information, but rather blurring the information provided by sensors closer to the fire and thus making the overall estimation process less accurate.

\subsection{Alternative sensors}

In addition to the invariants related to fire growth, other model parameters of interest can be estimated as well. Soot production in fires is a topic of prime importance, and state-of-the-art fire models cannot accurately predict the soot yield for most cases ${ }^{32}$. In FDSv5 the soot yield is not computed as a result of flame kinetcis, but is an input parameter for the simulation. Thus, the estimation of a global soot yield as the invariant alongside with the spread rate is studied for this case. A true value of 0.015 is used to generate the synthetic data.

Figure 13 shows that while the spread rate can be estimated correctly after 4 iterations, the soot yield cannot be obtained by assimilating temperature data. The sensitivity of the simulated temperature distribution to the soot yield is much lower than the sensitivity to the

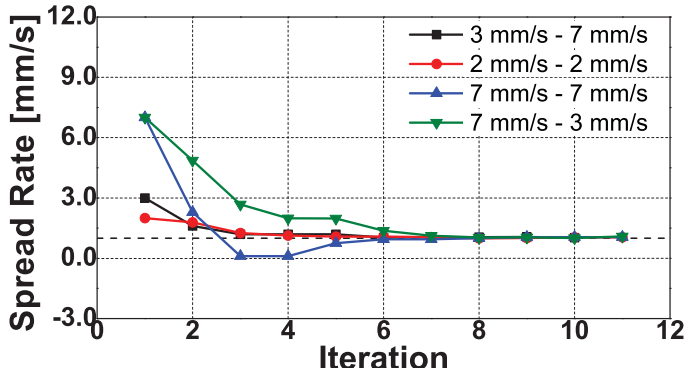

(a)

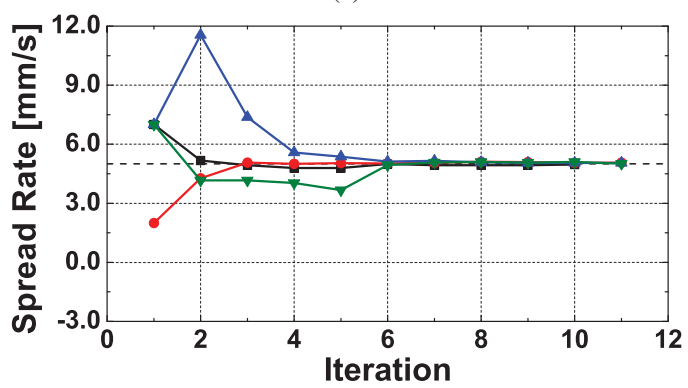

(b)

Figure 12: Estimation of spread rates for two burning items using sensors close to the fire and discarding sensors far away from the fire location; a) shows the convergence of the spread rate on the bed, and b) shows the spread rate of the wall fire. The dashed line marks true value.

spread rate, and therefore the temperature data alone does not allow for distinguishing between different soot yields in conjunction with the spread rate.

Other sensor data will have to be assimilated in order to estimate the soot yield. Beam detectors are widely used as smoke detectors in industrial facilities. They consist of a laser emitter on one side, and a receiver on the other side across the compartment. The incoming light at the receiver is compared to the intensity emitted, and a percentage of obscuration is calculated. When a certain threshold is passed due to obscuration of smoke, an alarm is triggered. Although beam detectors are currently only used in a binary mode (fire/no fire), it is possible to extract a continous signal of obscuration.

The previous test case is repeated estimating the spread rate of a single burning fire alongside the soot yield of the fire. This time, instead of the ceiling temperature, obscuration observations alone are assimilated into the model. The location of the beam detectors is indicated in fig. 5 .

Figure 14 shows the convergence behaviour for the spread rate and the soot yield. The obscuration due to the smoke of the fire provides enough information for 


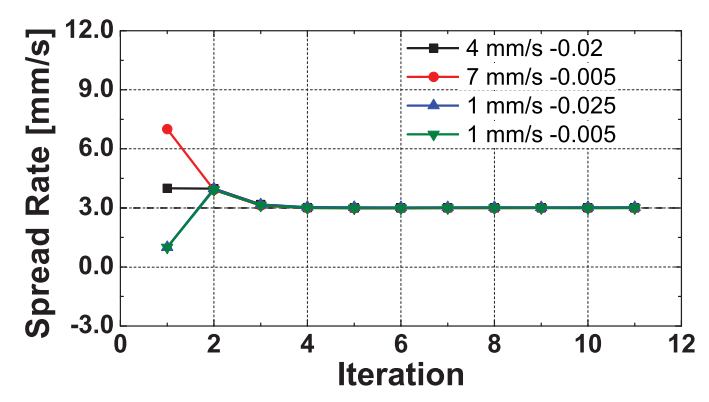

(a)

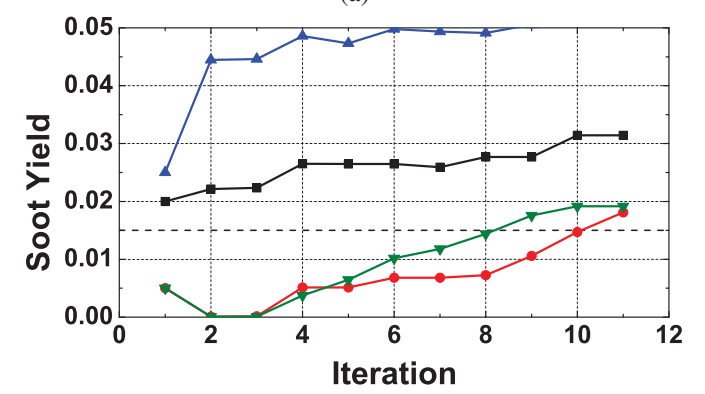

(b)

Figure 13: Estimation of the spread rate and soot yield of a single burning item; a) shows the convergence of the spread rate, and b) shows the soot yield. The dashed line marks the true value.

the accurate estimation of these two parameters.

When adding the fuel burning rate as a parameter to estimate, assimilation of the obscuration data alone could not pick up the joint contributions of spread rate and fuel burning rate to the overall HRR. For the full characterization of a compartment fire, including spread rate, fuel burning rate and the soot production, the beam detectors were therefore used in conjunction with thermocouples in the ceiling.

The thermocouple data together with the obscuration provided the necessary information for the estimation of all three parameters, as shown in fig. 15. The spread rate (fig. 15a) and the soot yield of the fire (fig. 15c) are estimated with acceptable accuracy $(<10 \%)$ after four to five iterations. Convergence of the fuel mass flow rate (fig. 15b) can take more than six iterations for certain combinations of first guesses.

\section{Towards positive lead times}

CFD based forecasts, as opposed to forecasts based on simpler fire models (e.g. zone models), provide spatial resolution. However, while positive lead times are possible using simple models ${ }^{8}$, this can at the present not be achieved using CFD. The goal of this paper is to

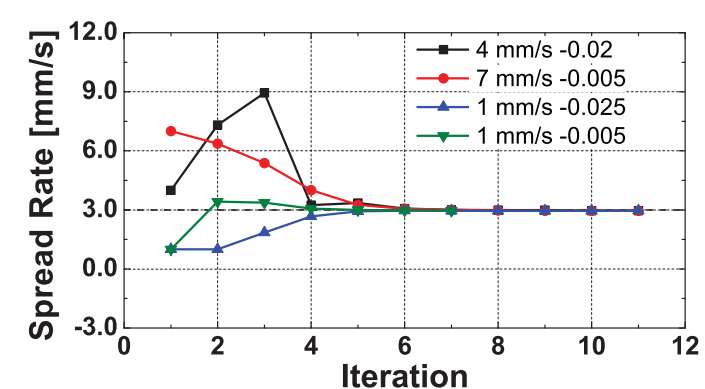

(a)

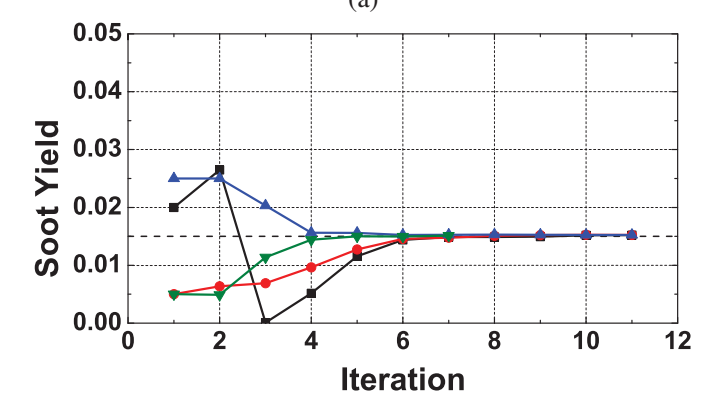

(b)

Figure 14: Estimation of spread rate and soot yield of a single burning item using beam detectors as observations for comparison. Graph a) shows the convergence of the spread rate; b) shows the convergence of the soot yield. The dashed line marks the true value.

illustrate that when observations from the fire are assimilated into the model, it is possible to reduce computation time considerably without losing accuracy. The use of High Performance Computing techniques ${ }^{3}$ can accelerate further the inverse problem and thus increase the lead times.

The lead times presented in the following sections refer to the time between the end of the assimilation window and the time where the forecast diverges $>10 \%$ from the observed fire development. The assimilation time (i.e. the time it takes to run several iterations of forward model in the optimisation and re-run it to make a forecast) is not considered here. It would not be possible to obtain positive lead times considering these additional times in spite of the greatly reduced computation time. In the previous sections the estimation of invariant parameters is demonstrated (which corresponds to the assimilation of data). Generally it takes six to eight iterations of the optimization cycle to obtain convergence. Each iteration involves many parallel executions of the CFD model (in this case FDSv5), so that each iteration lasts as long as it takes to run the slowest of the parallel CFD runs. Bearing in mind that a detailed CFD model of a compartment fire can take several hours and even 


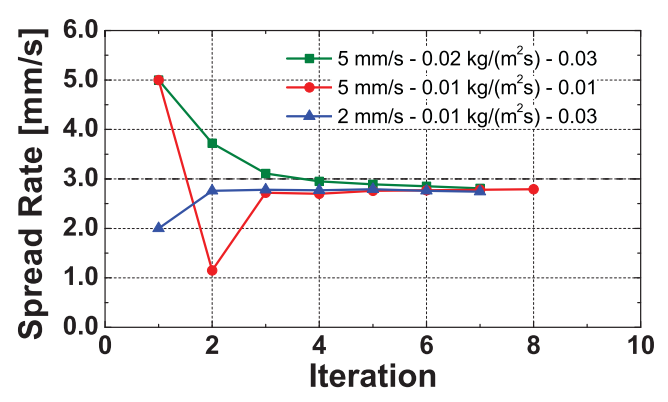

(a)

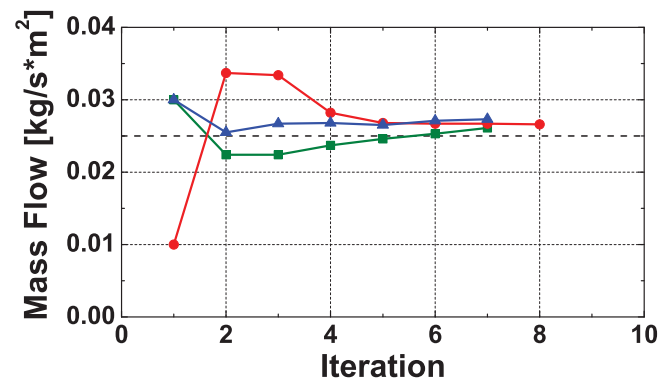

(b)

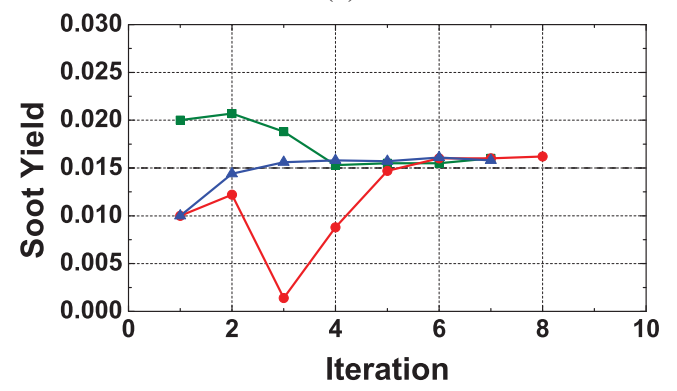

(c)

Figure 15: Estimation of three invariants for a single item burning. Graph a) shows the convergence of the spread rate. b) shows the convergence of the fuel mass flow rate, and c) shows the estimation of the soot yield of the fire. The dashed line marks the true value.

days to run, it would not be practical to use them for forecasting, as the lead time will be negative (i.e. the prediction arrives after the event has taken place).

However, since sensor data are assimilated to steer the simulations, this can compensate for a simpler and faster forward model. The observations from the fire provide the information lost by the approximations in a simpler model. Computations can thus be accelerated considerably using larger grid sizes. For example, a grid size of $25 \mathrm{~cm}$ speeds up the calculations over 100 times compared to grid sizes of $5 \mathrm{~cm}$.

The methodology presented in section 6 is independent of the size of grid of the forward model, as long as it represents the flow field and temperature profiles within acceptable bounds. The CFD code (FDSv5) has been tested for different grid sizes, and it has been established that for a grid size of $25 \mathrm{~cm}$, temperature profiles are still in good agreement with simulations done with grid sizes of $5 \mathrm{~cm}$ edges (compare ${ }^{21}$ ). It was thus decided to study the case with a $25 \mathrm{~cm}$ grid size for the forward model in the following forecast.

The same fire scenario as presented in fig. 4 was used to forecast fire growth. The geometry of the compartment and fuel type, location and origin are assumed known. A coarse grid of $25 \mathrm{~cm}$ was used in the forward model. Note that a fine grid of $5 \mathrm{~cm}$ was used to generate the synthetic observations. The true spread rate was $5 \mathrm{~mm} / \mathrm{s}$, which corresponds to a medium to fast fire typical in mattresses ${ }^{1}$.

The full set of temperature and beam sensors shown in fig. 5 was used, but instead of ceiling wall temperatures as used in the previously, gas temperatures close to the ceiling $(5 \mathrm{~cm})$ were used for assimilation into the model in this case. This is changed because in a CFD model temperatures are calculated in the center of each grid cell, representing thus the average temperature of the whole cell. This can lead to important underpredictions of the temperature close to the ceiling if the cell size is larger then the depth of the ceiling jet, resulting in an underprediction of the heat fluxes to the ceiling and a subsequent underprediction of the wall temperature.

The grid size of $25 \mathrm{~cm}$ is larger than the typical depth of the ceiling jet $(<20 \mathrm{~cm})$. The ceiling jet is formed when the vertical fire plume impinges on the ceiling surface and deflects the smoke horizontally towards the compartment walls. In order to overcome the underprediction due to average temperatures in the forecast model, observations were taken inside and below the ceiling jet in the true fire, and an average of both was used for assimilation.

Finding the optimal width of the assimilation window requires some analysis, since it has to be sufficiently large as to gather enough data, but small enough to still leave time to make a forecast ${ }^{8}$.

Figure 16 shows the estimated spread rate of the fire as a function of the width of the assimilation window. With $150 \mathrm{~s}$ worth of data the spread rate is estimated to be $5.63 \mathrm{~mm} / \mathrm{s}, 13 \%$ higher than the spread rate of the true fire. As more data come in a better estimation can be made, and after $330 \mathrm{~s}$ worth of data the estimated spread rate is within $1 \%$ of the true spread rate.

Figure 17 shows the forecast made with $150 \mathrm{~s}$ of data. The coarse grid simulation used for the assimilation cycle is not able to pick up the early fire growth correctly, producing an overprediction of the spread rate. As a 


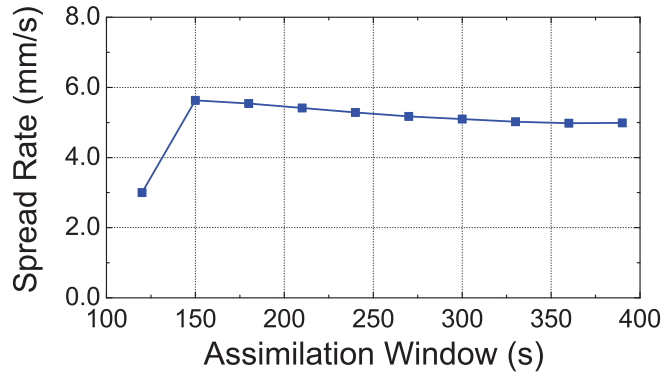

Figure 16: Spread rate as a function of the growing assimilation window.

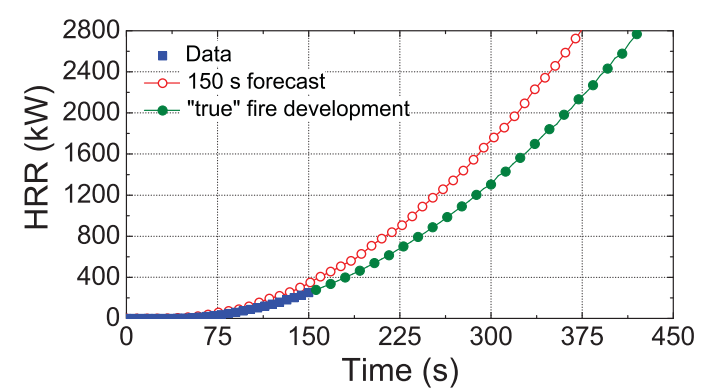

(a)

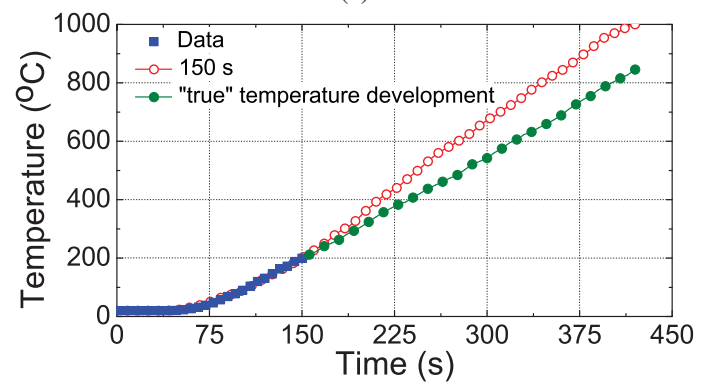

(b)

Figure 17: Forecast after $150 \mathrm{~s}$ of assimilated data. The observed data is shown together with the future development and the forecast of the fire; a) HRR b) Average temperature of the ceiling jet.

consequence of this the HRR is overpredicted (fig. 17a), which results in forecasted temperatures higher by $20 \%$ than the true fire development (fig. 17b). The lead time, defined as the time ahead of the event during which the forecasted temperature is less than $15 \%$ off the true temperature, is only approximately $50 \mathrm{~s}$.

Figure 18 shows the forecast made with $330 \mathrm{~s}$ worth of data. The HRR is in very good agreement with the true fire (fig. 18a), and although forecasted temperatures are slightly higher than the true fire development (9\%), the slope of the temperature curve is very similar to the true fire development (fig. 18b). The lead time in this

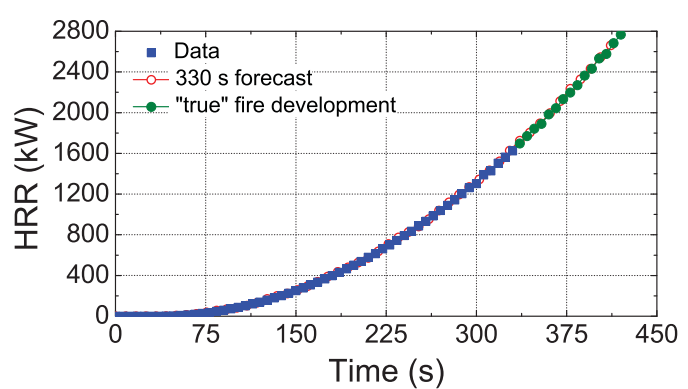

(a)

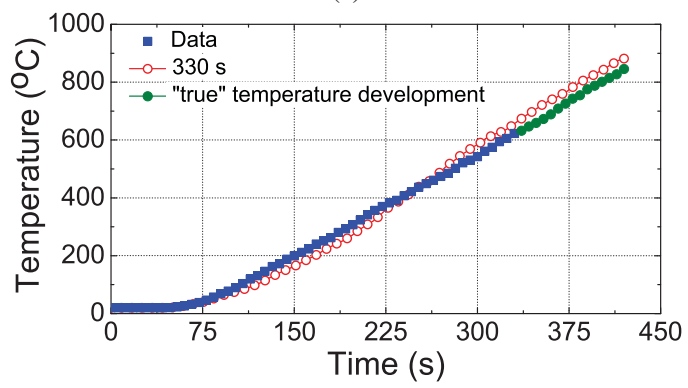

(b)

Figure 18: Forecast after $330 \mathrm{~s}$ of assimilated data. The observed data is shown together with the future development and the forecast of the fire; a) HRR b) Average Ceiling Jet temperature.

case is $120 \mathrm{~s}$ or when the next critical event changing the spread behaviour takes place.

The assimilation and forecast cycle using $330 \mathrm{~s}$ of data took around 10 min on a standard dual core PC. If a blind prediction of the same fire scenario (without feedback from the fire) were to be made, it would require a grid size $<1 \mathrm{~cm}$ in order to be able to reproduce the underlying physics with enough accuracy. Such a simulation would take several weeks to run (compare ${ }^{5}$ ).

\subsection{Unknown fuel source}

Although it is realistic to assume that the geometry of the room (walls, windows, doors) is known, the location and dimensions of the fuel sources in buildings typically changes widely and unexpectedly. Desks, chairs, bed and other potential fire loads can be moved freely within the compartment, and their composition and layout at the moment of a fire event are impossible to establish a priori. The fire origin is relatively easy to identify. As a first order approximation it would be enough to select the location of the thermocouple with the highest temperature reading. Thus, it is realistic to assume that it is known. But the forecast has to be robust regarding different locations and dimensions of the fuel sources. This is investigated here. 
The true fire involves a single item burning, the bed, and this is ignited at a corner and spreads radially (true spread rate of $3 \mathrm{~mm} / \mathrm{s}$ ). The forward model assumes a fire ignited on the middle of the wall and a fuel surface covering all the area of the compartment, although at an elevated level ( $0.4 \mathrm{~m}$ above the floor). This will obviously lead to a larger fire size than in the true fire after the spread has reached the bed edges, and a subsequent divergence of the forecast, as in the forward model the fire growth is only limited by the walls of the compartment.

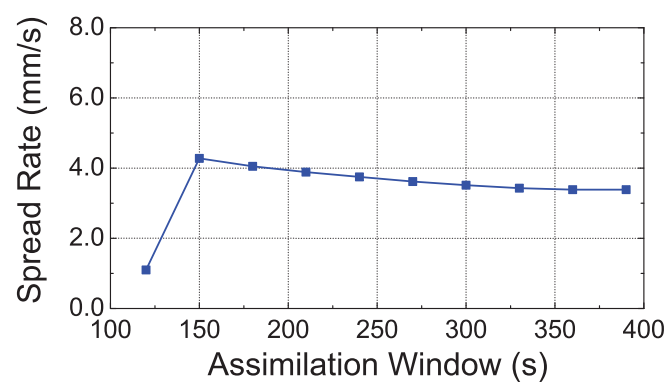

Figure 19: Spread rate for an unknown fuel load as a function of the assimilation window width.

Figure 19 shows the estimated spread rate of the fire as a function of the width of the assimilation window. The estimated spread rate is much smaller than the true spread rate. This is due to the layout of the true fire, which ignites at the corner of the bed, and the fire area starts growing as the quarter of a circle. In the forward model, without prior knowledge of the fuel source, the fire does not ignite at a corner (although still at the wall) and can grow as a half-circle. For the same fire area the forward model without prior knowledge of the fuel has thus a smaller radius (i.e. smaller spread rate).

Figure 20 shows the forecast made with $330 \mathrm{~s}$ worth of data. Although the HRR is underpredicted slightly $(<$ $15 \%$ ) (fig. 20a), the temperatures are in good agreement with the temperature observations $(<13 \%$, fig. 18b). Note that temperatures are only recorded close to the ceiling, and that the lower HRR leading to higher temperatures as shown in fig. 20 is thus not necessarily a contradiction. This is the result of a different smoke flow pattern in the forward model than in the true fire due to the different fire areas which affects entrainment and the smoke plume dynamics ${ }^{20}$. Using sensors that are not only in the ceiling jet region would improve the forecast.

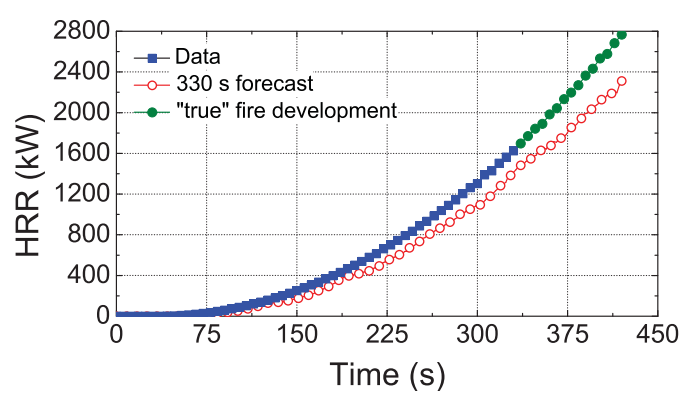

(a)

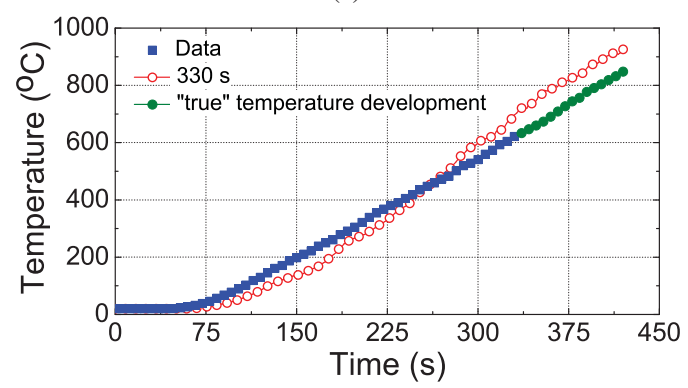

(b)

Figure 20: Forecast after $330 \mathrm{~s}$ of assimilated data with no prior knowledge of the fuel load. The observed data is shown together with the future development and the forecast of the fire; a) HRR b) Average Ceiling Jet temperature.

\section{Conclusions}

The state-of-the-art of CFD in fire dynamics is not fast enough or accurate enough to provide valid forecasts. The main problem is in the complex heat and a mass coupling between the flames and the pyrolysing fuel. This is solved here by proposing a simple fire growth model which captures in a few invariant parameters the governing dynamics. Then, sensor observations are assimilated by posing an inverse problem.

A series of real-scale compartment fire cases are investigated using the CFD code FDSv5. The different parameters were estimated with a reasonable computational effort. Up to three different invariant are considered (spread rate, burning rate and soot yield) in scenarios with one or two fires and different origins. It is shown that the methodology does not require an exact knowledge of the fuel layout in the compartment.

The effect of density, location, cover area and type of sensors is studied. Forecast quality grows with the density and heterogeneity of the sensor set. Larger cover areas do not always lead to better forecast as data from no-fire areas decrease the forecast quality.

The results proof that the use of sensor data compen- 
sates fine model details that are lost as a consequence of simplification and coarse grids, and allows for acceleration. Although actual positive lead times are not reached here, it is shown that the use of relatively coarse grid size in the forward model significantly accelerates the assimilation (up to two orders of magnitude) without loss of forecast accuracy. Actual positive lead times with CFD are possible by reducing the computational time by at least another order of magnitude in the near future using high performance computing techniques. These results are a fundamental step towards the development of a forecast technology able to lead the fire emergency response.

\section{Acknowledgements}

The support for W. Jahn from the European Union Programme AlBan of High Level Scholarships for Latin America (Grant No. E06D100038CL) and BRE Trust is gratefully acknowledged. This work forms part of FireGrid, www.firegrid.org, and has been partially funded by the UK Technology Strategy Boards Collaborative Research and Development Programme. Our gratitude to the Building and Fire Research Laboratory at NIST for developing the computer fire model FDS and making it available.

[1] Drysdale, D.. An Introduction to Fire Dynamics. ISBN 0-47031903-8; New York: Wiley \& Sons; $3^{\text {rd }}$ ed.; 2011.

[2] Cowlard, A., Jahn, W., Abecassis-Empis, C., Rein, G., Torero, J.. Sensor Assisted Fire Fighting. Fire Technology 2010;46(3):719-741. DOI:10.1007/s10694-008-0069-1.

[3] Upadhyay, R., Pringle, G., Beckett, G., Potter, S., Han, S., Welch, S., et al. An Architecture for an Integrated Fire Emergency Response System for the Built Environment. Fire Safety Science 2008;9:427-438. DOI:10.3801/IAFSS.FSS.9427, http://www.era.lib.ed.ac.uk/handle/1842/2703.

[4] Rein, G., Torero, J., Jahn, W., Stern-Gottfried, J., Ryder, N., Desanghere, S., et al. RoundRobin Study of a priori Modelling Predictions of the Dalmarnock Fire Test One. Fire Safety Journal 2009;44(4):590-602. DOI:10.1016/j.firesaf.2008.12.008, http://www.era.lib.ed.ac.uk/handle/1842/2704.

[5] Kwon, J., Dembsey, N., Lautenberger, C.. Evaluation of FDS v.4: Upward Flame Spread. Fire Technology 2007;43(4):255284. DOI:10.1007/s10694-007-0020-x.

[6] Lorenz, E.. Deterministic Nonperiodic Flow. Journal of the Atmospheric Sciences 1963;20(2):130-141.

[7] Cowlard, A., Auersperg, L., Richon, J., Rein, G., Welch, S., Usmani, A., et al. A Simple Methodology for Sensor Driven Prediction of Upward Flame Spread. Turkish Journal of Engineering and Environmental Sciences 2007;31(6):403-413.

[8] Jahn, W., Rein, G., Torero, J.. Forecasting fire growth using an inverse zone modelling approach. Fire Safety Journal 2011;46(3):81-88. DOI:10.1016/j.firesaf.2010.10.001.

[9] Jahn, W.. Inverse Modelling to Forecast Enclosure Fire Dynamics. Ph.D. thesis; The University of Edinburgh; 2010. Http://www.era.lib.ed.ac.uk/handle/1842/3418.
[10] Kalnay, E.. Atmospheric Modeling, Data Assimilation and Predictability. ISBN 978-0-521-79629-3; Cambridge: Cambridge University Press; $1^{\text {st }}$ ed.; 2003.

[11] Schlatter, T.. Variational assimilation of meteorological observations in the lower atmosphere: a tutorial on how it works. Journal of Atmospheric and Solar-Terrestial Physics 2000;62(17):1057-1070.

[12] Hoke, J., Anthes, R.. The Initialization of Numerical Models by a Dynamic-Initialization Technique. Monthly Weather Review 1976;104(12):1551-1556.

[13] Harms, D., Raman, S., Madala, R.. An Examination of Four-Dimensional Data Assimilation Techniques for Numerical Weather Prediction. Bulletin Amercian Meteorolical Society 1992;73(4):425-440.

[14] Richards, R., Munk, B., Plumb, O.. Fire Detection, Location and Heat Release Rate Trough Inverse Problem Solution. Part I: Theory. Fire Safety Journal 1997;28(4):323-350. DOI:10.1016/S0379-7112(97)00005-2.

[15] Leblanc, M., Trouvé, A.. Inverse Modeling of Enclosure Fire Dynamics. Proceedings of the $6^{\text {th }}$ U.S. national combustion meeting; 2009.

[16] Koo, S., Fraser-Mitchel, J., Welch, S.. Sensor-steered fire simulation. Fire Safety Journal 2010;45(3):193-205. DOI:10.1016/j.firesaf.2010.02.003.

[17] Jahn, W., Rein, G., Torero, J.. Forecasting fire growth using an inverse CFD modelling approach in a real-scale fire test. Fire Safety Science 2011;10(in press).

[18] Baum, R., Ezekoye, A., McGrattan, K., Rehm, R.. Mathematical Modeling and Computer Simulation of Fire Phenomena. Theoretical and Computational Fluid Dynamics 1994;6(23):125-139.

[19] McGrattan, K., Rehm, R., Baum, H.. Large Eddy Simulations of Smoke Movement. Fire Safety Journal 1998;30(2):161-178.

[20] Jahn, W., Rein, G., Torero, J.. The Effect of Model Parameters on the Simulation of Fire Dynamics. Fire Safety Science 2008;9:1341-1352. DOI:10.3801/IAFSS.FSS.9-1341, http://www.era.lib.ed.ac.uk/handle/1842/2696.

[21] Jahn, W., Rein, G., Torero, J.. A posteriori modelling of the growth phase of Dalmarnock Fire Test One. Building and Environment 2011;46(5):1065-1073. DOI:10.1016/j.buildenv.2010.11.001.

[22] Forkel, H., Janicka, J.. Large-Eddy Simulation of a Turbulent Hydrogen Diffusion Flame. Flow, Turbulence and Combustion 2000;65(2):163-175. DOI:10.1023/A:1011497715385.

[23] Xie, W., DesJardin, P.. An embedded upward flame spread model using 2D direct numerical simulations. Combustion and Flame 2009;156(2):522-530. DOI:10.1016/j.combustflame.2008.11.011.

[24] Fernandez-Pello, C.. The Solid Phase. In: Cox, G., editor. Combustion Fundamentals of Fire; chap. 2. Academic Press Ltd.; 1995, p. 31-100.

[25] Cooper, L.. Compartment Fire-Generated Environment and Smoke Filling. In: DiNenno, P., editor. SFPE Handbook of Fire Protection Engineering; chap. 3-10. Quincy, MA 02269: National Fire Protection Association; 3 ed.; 2002, p. 3-243-3267.

[26] Sivanandam, S., Deepa, S.. Introduction to Genetic Algorithms. ISBN 978-3-540-73189-4; Berlin, Heidelberg: Springer Verlag; $1^{\text {st }}$ ed.; 2008.

[27] Nocedal, J., Wright, S.. Numerical Optimization. ISBN 9780387-30303-1; USA: Springer; $2^{\text {nd }}$ ed.; 2006.

[28] Bouttier, F., Courtier, P.. Data Assimilation concepts and methods. Tech. Rep.; European Centre for Medium-Range Weather Forecasts; 2001.

[29] McGrattan, K., Klein, B., Hostikka, S., Floyd, J.. Fire Dy- 
namics Simulator (Version 5) - User's Guide. NIST Special Publication 1019-5; 2008

[30] Babrauskas, V.. Heat Release Rates. In: DiNenno, P., editor. SFPE Handbook of Fire Protection Engineering; chap. 3-1. Quincy, MA 02269: National Fire Protection Association; 3 ed.; 2002, p. 3-1-3-37.

[31] Cowlard, A.. Sensor and Model Integration for the Rapid Prediction of Concurrent Flow Flame Spread. Ph.D. thesis; The University of Edinburgh; 2009. Http://www.era.lib.ed.ac.uk/handle/1842/2753.

[32] Moss, J., Stewart, C., Young, K.. Modelling Soot Formation and Burnout in a High Temperature Laminar Diffusion Flame Buning under Oxygen-Enriched Conditions. Combustion and Flame 1995;101(4):491-500. DOI:10.1016/00102180(94)00233-I. 